\title{
Study of Phenomenon STT (Spin Transfer Torque) on Permalloy NiFe Material Shaped Nanowire Using Micromagnetic Simulation
}

\author{
Khiptiatun Ni'mah ${ }^{1}$, Lutfi Rohman ${ }^{1, a}$ and Endhah Purwandari ${ }^{1}$ \\ ${ }^{1}$ Department of Physics, Faculty of Mathematics and Natural Sciences, University of Jember, \\ Jalan Kalimantan No. 37, Jember 68121, Indonesia \\ ael_rahman.fmipa@unej.ac.id
}

\begin{abstract}
STT is a process of controlling the spin currents in spintronic. This simulation aims to know the properties of NiFe permalloy materials' properties by studying STT phenomenon-shaped nanowire that can be applied in storage devices, like MRAM. The material's magnetic properties include magnetization value, energy in the ferromagnetic system, and the speed of the domain wall movement, obtained by injecting the electric current density through a micro magnetic simulation using the NMAG program. This simulation's result is that the domain wall's position will shift faster along the nanowire when we inject current density to the nanowire. Current density injection will produce a domain wall pressure on the domain structure, resulting in a change in the material's magnetization value. The graph of magnetization relation to time $(\mathrm{M}-\mathrm{t})$, shown along with the increasing electric current density, we obtain oscillation magnetization change will increase. The larger the given diameter, the total energy generated will increase, demagnetization energy tends to be greater than the energy exchange. The greater the polarization of the material provided at the same diameter, the speed of the domain wall movement will be greater too.
\end{abstract}

Keywords: Spintronic, STT, Permalloy NiFe, Material Polarization, Domain Wall Speed, Magnetization

\section{Introduction}

Spintronic is a device that works by exploiting changes in the electron spin configuration to control the movement of charge carriers [1]. The advantages of spintronic are its fast data processing capability, smaller device size, and low energy consumption [2]. One of the potential materials for this spintronic device application is a ferromagnetic material. The ferromagnetic materials have spontaneous magnetization or what is commonly known as a magnetic domain [3]. $\mathrm{Ni}_{80} \mathrm{Fe}_{20}$ ferromagnetic material or permalloy material is a magnetic alloy material or metal alloy material composed of $80 \%$ nickel and $20 \%$ iron. Permalloys with a higher nickel content than iron tend to have greater permeability than low nickel permalloys. Permalloys are sensitive to impact, and their magnetism is strongly influenced by pressure [4].

Research on thin films' magnetic properties and magnetic domain-walls in ferromagnetic materials has attracted scientists' attention in the last two decades. That is because these studies have the potential for data storage media based on magnets (magnetic memory devices) and magnetic sensors that carry out by utilizing the configuration of magnetic moments in the magnetic domain (magnetic domain) and magnetic domain wall (magnetic domain wall). [2]. In general, magnetic domain research can divide into two parts: research related to the magnetic domain structure and the domain-wall dynamics in nanowire-shaped ferromagnetic materials. Applying an external magnetic field [5] or injection of a current polarized (current polarized) known as the STT (Spin Transfer Torque) [6]. 


\section{Theoretical Background}

STT is a process of controlling spin currents in the spintronics field [2]. The ferromagnetic material injected with the current will change the spin direction orientation known as Spin Transfer Torque. It will move the domain walls accompanied by a change in spin direction [7]. Movement of the walls domain in the direction of current flow can generate when the current polarizes the spins. The application of this current can produce pressure in the domain's walls in an electron flow direction. The domain's wall pressure will make a magnetization change as the current density increases [8]. The dynamic in spin orientation is of particular interest in the development of magnetic storage devices. STT can generate when the material is injected with an electric current to change the spin orientation at the electron magnetic dipole moment [9].

\section{Materials and Methods}

This micromagnetic simulation program uses the Finite Element (NMAG) method. NMAG is an open-source program that in the Linux operating system. The program has a Python script (.py), an input containing material parameters executed in the Linux terminal. The basic concept of the Finite Element method is to solve a problem by dividing the object of analysis into finite small parts. These little pieces are then analyzed, and the results are recombined to find a solution for the whole area. The use of this Finite Element method can solve LLG problems from a system by converting it to a discrete system so that it is possible to solve it numerically. This micromagnetic simulation is run based on the LLG equation as follows:

$\frac{\partial \mathrm{M}}{\mathrm{dt}}=\gamma \mathrm{M} \times \mathrm{H}_{\mathrm{eff}}-\frac{\gamma \alpha}{\mathrm{M}_{\mathrm{g}}} \mathrm{M} \times\left(\mathrm{M} \times \mathrm{H}_{\mathrm{eff}}\right)-\alpha^{2} \frac{\partial \mathrm{M}}{\mathrm{dt}}$

The variation of the electric current density represented in this simulation is $5 \times 10^{10}, 10 \times 10^{10}$, $15 \times 10^{10}, 20 \times 10^{10} \mathrm{~A} / \mathrm{m}^{2}$. Nanowire diameter variations to be simulated are $10 \mathrm{~nm}$ and $20 \mathrm{~nm}$. The variation of the polarization of the material given is equal to 0.5 and 1 . The table shows the input data used in the micromagnetic simulation of the NiFe permalloy material Table 1 . The data includes Ms (saturation magnetization), K (anisotropy constant), A (exchange constant), and damping constant.

Table 1. Parameter of NiFe Permalloy material

\begin{tabular}{cccc}
\hline $\mathrm{Ms}(\mathrm{A} / \mathrm{m})$ & $\mathrm{K}(\mathrm{J} / \mathrm{m})$ & $\mathrm{A}\left(\mathrm{J} / \mathrm{m}^{3}\right)$ & Damping Constant \\
\hline $800 \times 10^{3}$ & $5 \times 10^{3}$ & $1,3 \times 10^{-11}$ & 0,5 \\
\hline
\end{tabular}

The parameters which have to enter into the script file are (.geo), which contains the diameter and length of the nanowire, and (.py), which is a variation of the electric current density injection and a variation of polarization material. The next step is simulating Linux Terminal. The simulation results are in the form of numerical data consisting of files (.ndt) and (.vtk), which will be used in the visualization stage. Visualization of domain images is done using mayavi software through files (.vtk). The next step is to create a graph plotted from the file (.ndt) using the Origin software for each parameter that has been varied. The visualization and graph plot results will later be used for the analysis or interpretation of the results. After the analysis (images, charts, and tables) is carried out, a conclusion will be obtained, indicating that this research has been completed. 


\section{Results and Discussion}

\section{The Effect of Injection Electric Current Density Variations on the Magnetization of Nanowire-Shaped NiFe Permalloy Materials on the STT (Spin Transfer Torque) Phenomenon}

Observation of the STT phenomenon due to the effect of electric current density injection on the magnetization of materials is carried out in three stages, namely observing the position of the domain wall, the structure of the domain wall due to the injection of electric current density in each nanowire diameter and the polarization of different materials and analyzing the graph of the relationship between magnetization and time $(\mathrm{M}-\mathrm{t})$. The first stage in studying the STT phenomenon is observing the domain wall's position due to variations in electric current density. The position of the domain wall was observed by visualization. The result of isometric direction to nanowire length $100 \mathrm{~mm}$, diameter $10 \mathrm{~nm}$, and $20 \mathrm{~mm}$ was injected by an electric current density of $5 \times, 10^{10}, 10 \times 10^{10}, 15 \times 10^{10}$, and $20 \times 10^{10} \mathrm{~A}^{\mathrm{M} 2}$ when $\mathrm{t}=6 \mathrm{~ns}$ shown in Figures 1 and 2.
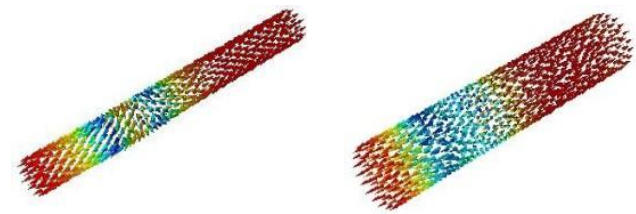

(a)
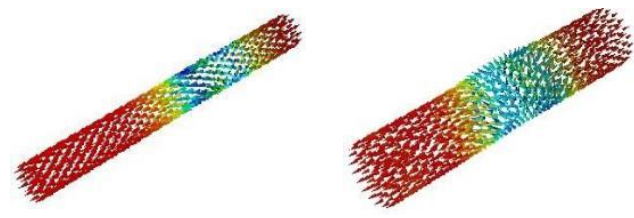

(c)

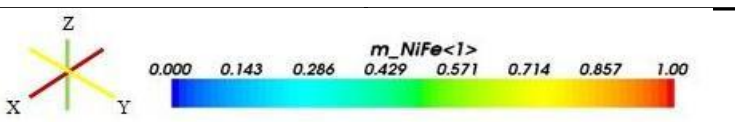

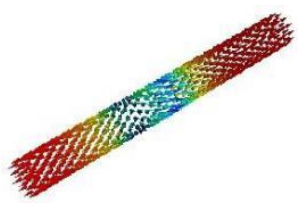

(b)

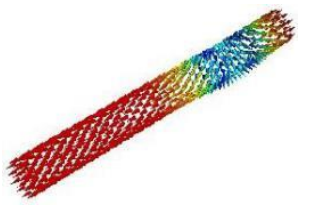

(d)

Figure 1. The shift in the position of the domain walls in the material permalloy NiFe with current density variations $5 \times 10^{10}, 10 \times 10^{10}, 15 \times 10^{10}$, and $20 \times 10^{10} \mathrm{~A} / \mathrm{m}^{2}$ when the polarization of the material is 1 

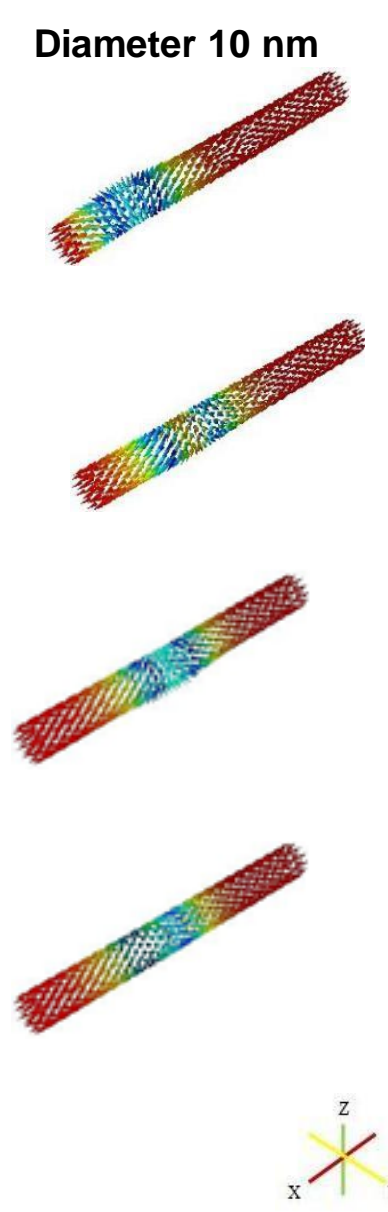

(d)

(a)

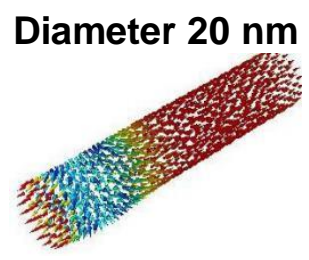

(b)
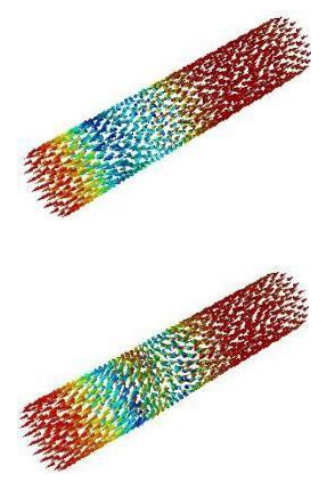

(c)

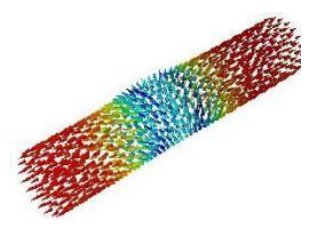

Figure 2. The shift in the position of the domain wall in the NiFe permalloy material with current density variations of $5 \times 10^{10}, 10 \times 10^{10}, 15 \times 10^{10}$, and $20 \times 10^{10} \mathrm{~A} / \mathrm{m}^{2}$ when the polarization of the material is 0.5

Figures 1 and 2 represent the position shift in the domain wall due to the influence of the variation of the electric current density injection in the NiFe permalloy material in the form of a nanowire when $\mathrm{t}=6 \mathrm{~ns}$. Based on Figure 4.1, with a material polarization of 1 at $10 \mathrm{~nm}$ and 20 $\mathrm{nm}$ in diameter, when the injected electric current density increases, the position of the domain wall will shift faster to reach the end of the nanowire so that the magnetization process that occurs in the nanowire will be faster. In this case, the magnetization value is indicated by the color index on the magnetization scale $\left(m_{-} \_\mathrm{NiFe}<1>\right.$ ). Under the previous study, which gives the more significant the electric current density, the magnetization process's faster will be [3].

The next STT phenomenon study observes the magnetic domain structure due to the injection of electric current density at each different diameter and polarization of materials. The change in the color index of the domain structure represents the scale of the magnetization value. Based on the domain wall's movement with a vortex structure that moves and rotates along the $x$-axis in the nanowire, the observed domain structure on the $z$-axis in each variation of the injection electric current density at $t=6$ ns can be seen in Figures 3 and 4 . 


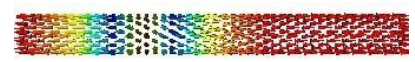

(a) $\mathrm{d}=10 \mathrm{~nm}$

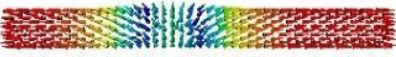

(b) $\mathrm{d}=10 \mathrm{~nm}$

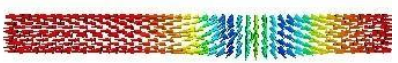

(c) $\mathrm{d}=10 \mathrm{~nm}$

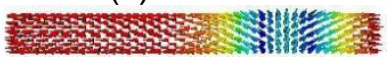

(d) $\mathrm{d}=10 \mathrm{~nm}$

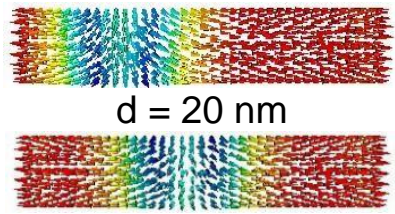

$\mathrm{d}=20 \mathrm{~nm}$

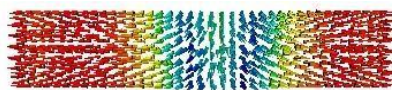

$\mathrm{d}=20 \mathrm{~nm}$

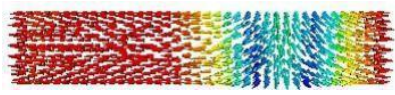

$\mathrm{d}=20 \mathrm{~nm}$

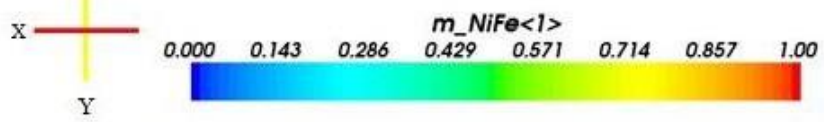

Figure 3. Domain wall structure on the z-axis in permalloy NiFe material with density Variation current $5 \times 10^{10}, 10 \times 10^{10}, 15 \times 10^{10}$, and $20 \times 10^{10} \mathrm{~A} / \mathrm{m}^{2}$ when polarizing the material 1

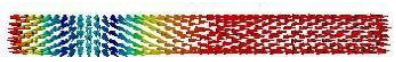

(a) $\mathrm{d}=10 \mathrm{~nm}$

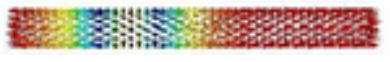

(b) $\mathrm{d}=10 \mathrm{~nm}$

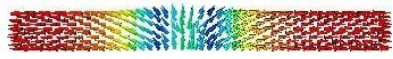

(c) $\mathrm{d}=10 \mathrm{~nm}$

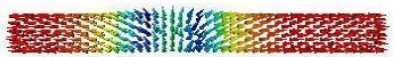

(d) $\mathrm{d}=10 \mathrm{~nm}$

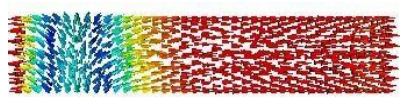

$\mathrm{d}=20 \mathrm{~nm}$

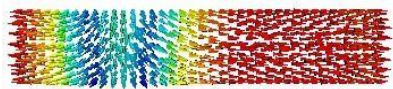

$\mathrm{d}=20 \mathrm{~nm}$

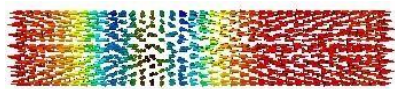

$\mathrm{d}=20 \mathrm{~nm}$

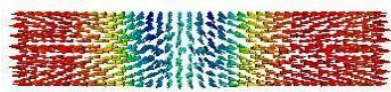

$\mathrm{d}=20 \mathrm{~nm}$

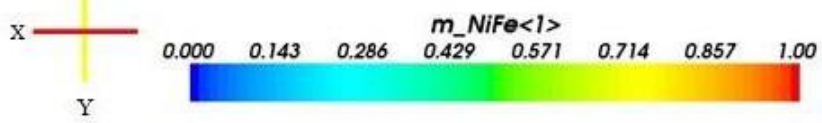

Figure 4. Domain wall structure on the $z$-axis in permalloyNiFe material with density variations currents $5 \times 10^{10}, 10 \times 10^{10}, 15 \times 10^{10}$, and $20 \times 10^{10} \mathrm{~A} / \mathrm{m}^{2}$ when polarizing the material is 0.5

Based on Figures 3 and 4, We can see the increase of the injected electric current density, and the polarization of the material current density can put pressure in the wall domain. The domain wall pressure in the domain structure can result in a change in the magnetization value. We can see in the domain wall structure whose magnetic value varies with each electric current density variation. The difference in the color index of the domain structure represents the scale of the magnetization value. A graph of magnetization supports the magnetization value change to time (M-t). 


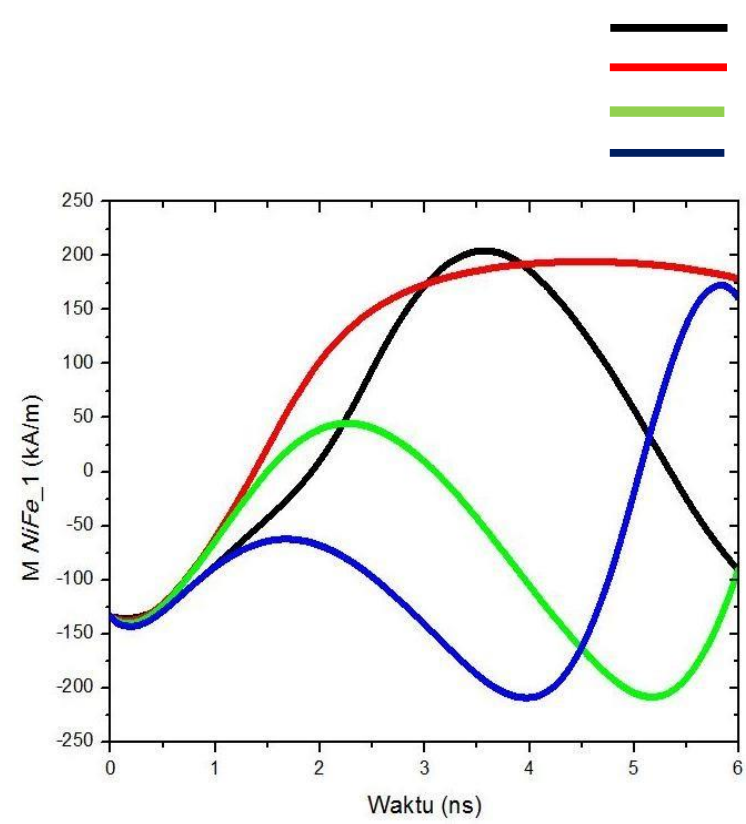

(a)
Current 5E10

Current 10E10

Current 15E10

Current 20E10

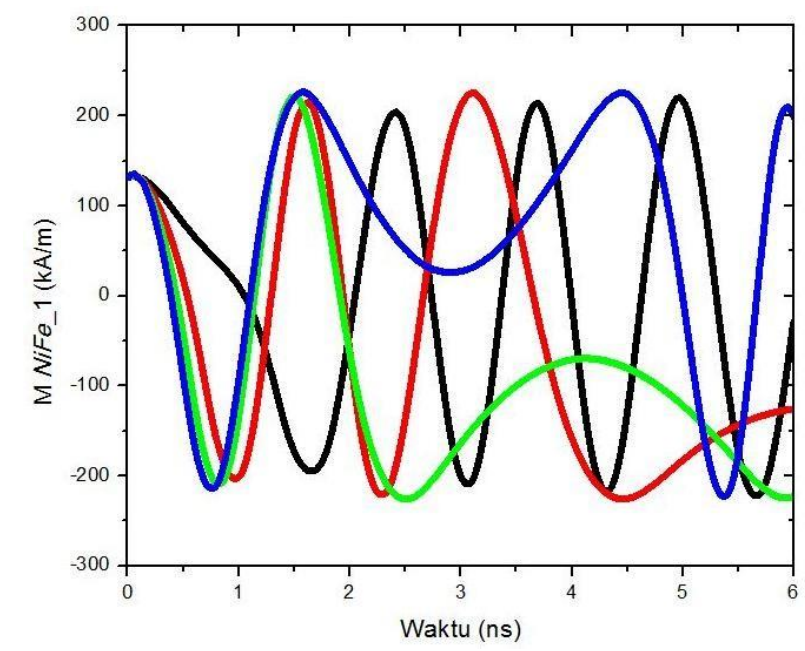

(b)

Figure 5. Graph of magnetization against time (M-t) for variations in electric current density on the polarization of material 1 with a nanowire diameter (a) $10 \mathrm{~nm}$ (b) $20 \mathrm{~nm}$

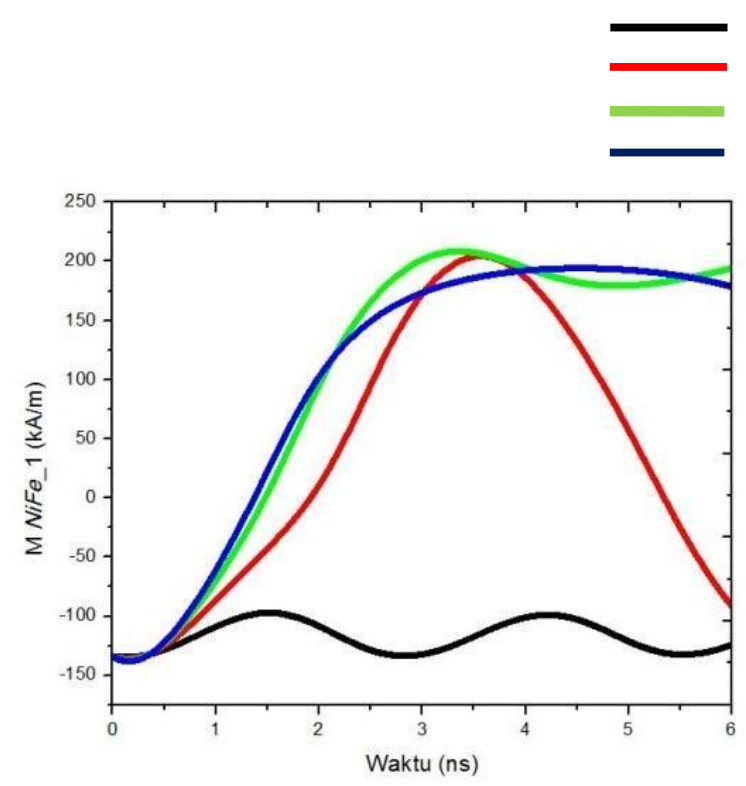

(a)
Current 5E10

Current 10E10

Current 15E10

Current 20E10

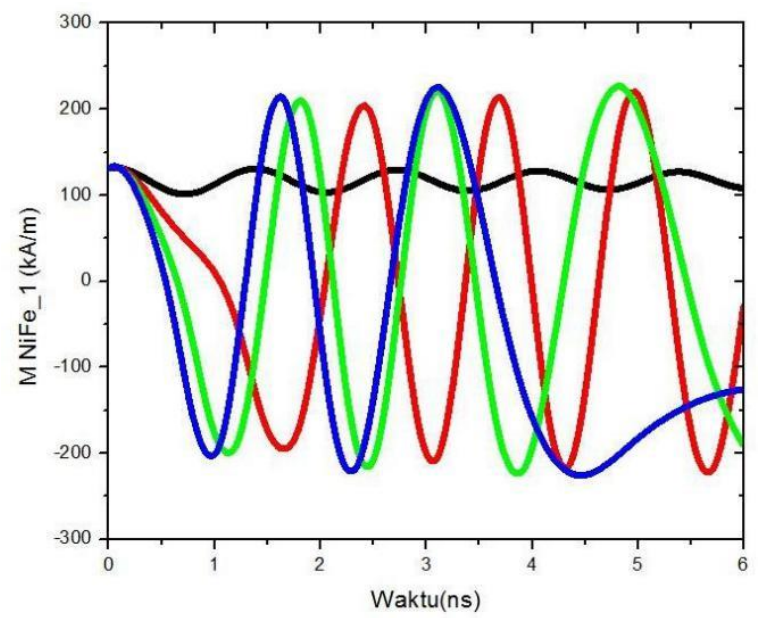

(b)

Figure 6. Graph of magnetization against time (M-t) for variations in electric current density on the polarization of material 0.5 with a nanowire diameter (a) $10 \mathrm{~nm}$ (b) $20 \mathrm{~nm}$

The magnetization value of the material will change with the injection of the electric current density. The change in the value of the magnetization of the material obtained is uncertain. 
Based on the magnetization graph in Figure $5(\mathrm{a})$, the $10 \mathrm{~nm}$ diameter of the oscillation is the tightest shown at the current density of $20 \times 10^{10} \mathrm{~A} / \mathrm{m}^{2}$ in blue, while in figure (b), the diameter of $20 \mathrm{~nm}$ is the densest oscillation at a current density of $5 \times 10^{10} \mathrm{~A} / \mathrm{m}^{2}$ with black. The magnetization graph in Figure 6 (a) is $10 \mathrm{~nm}$ in diameter and $20 \mathrm{~nm}$ of the densest oscillations occurring at a current of $5 \times 10^{10} \mathrm{~A} / \mathrm{m}^{2}$ with black color. The magnetizing oscillation density in the graph shows that the application of large electric currents requires less time to change the direction of spin [8]. Based on the overall analysis, we can see that the injected electric current density variation is very influential on the domain wall, the domain structure, and the magnetization graph against time (M-t). Following the theory that current injection will affect the magnetic domain, the magnetization value can produce a pressure domain wall in the direction of electron flow. The domain wall pressure will result in a change in magnetization as the current density increases [9].

\section{The Effect of Nanowire Diameter Variation on the Total Energy of Permalloy NiFe Materials on the STT (Spin Transfer Torque) Phenomenon}

This observation was carried to determine the effect of variations in the nanowire's diameter on NiFe permalloy material's total system energy. The energy contributing to this system includes total energy, demagnetization energy, and exchange energy in every electric current density. Analysis of the effect of nanowire diameter on energy through a graph of the energy relationship to electric current density plotted using Origin software when $t=6 \mathrm{~ns}$, as shown in Figure 7 .

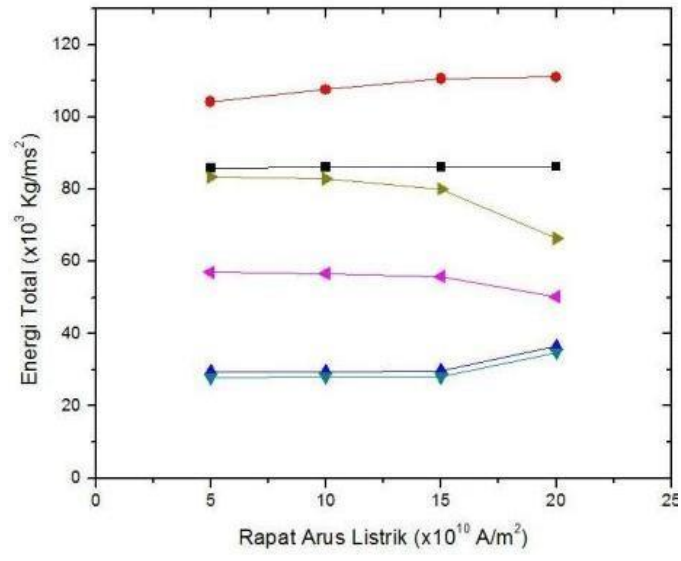

(a)

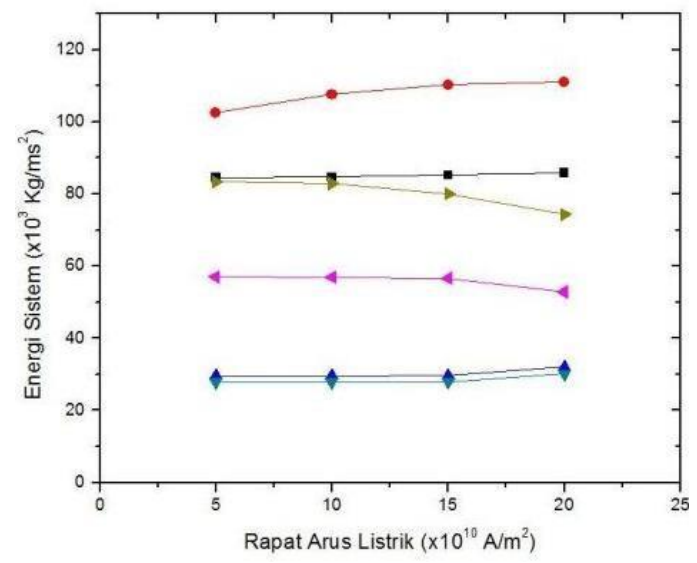

(b)

$$
\begin{aligned}
& \text { - Energi Total } 10 \mathrm{~nm} \\
& \text { - Energi Total } 20 \mathrm{~nm} \\
& \text { Energi Exchange } 10 \mathrm{~nm} \\
& - \text { Energi Exchange } 20 \mathrm{~nm} \\
& - \text { Energi Demagnetisasi } 10 \mathrm{~nm} \\
& - \text { Enenrgi Demagnetisasi } 20 \mathrm{~nm}
\end{aligned}
$$

Figure 7 . The graph of the relationship energy to electric current density on diameter 10 and $20 \mathrm{~mm}$ for polarization material (a) 1 and (b) 0,5

Terms of energy per unit volume represented system energy at different diameters in each variation of electric current density and material polarization. The first analysis that will be carried out on system energy is the analysis of total energy. The total energy produced will increase along with the increase in the injection electric current density. The total energy will 
also increase as the diameter of the nanowire varied gains. The exchange energy will increase along with the increase in the injection electric current density, but the energy will decrease along with the rise in the nanowire diameter. Along with the increase in the density of the injected electric current, the resulting demagnetization energy will decrease and increase when the nanowire diameter is varied.

The Effect of Material Polarization Variations on the Movement Speed of Domain Walls in Nanowire-Shaped NiFe Permalloys Resulting in the STT (Spin Transfer Torque) Phenomenon

Observation of the speed of movement of the domain wall in the NiFe permalloy material in the form of a nanowire due to the influence of variations in material polarization on the spin phenomenon of transfer torque is done using a time-resolved imaging method, which is to calculate the change in the position and time-domain wall represented in the corresponding image [10]. These results are described in the table form the mean Velocity of the domain walls' movement, as in Table 2.

Tabel 2. The average velocity of the movement of the domain walls when injected with an electric current density at a diameter of $10 \mathrm{~nm}$ and $20 \mathrm{~nm}$

\begin{tabular}{cccccc}
\hline \multirow{2}{*}{ No. } & \multirow{2}{*}{$\mathrm{J}\left(\mathrm{A} / \mathrm{m}^{2}\right)$} & \multicolumn{2}{c}{$\begin{array}{c}\text { Average Velocity }(\mathrm{m} / \mathrm{s}) \\
\mathrm{d}=10 \mathrm{~nm}\end{array}$} & \multicolumn{2}{c}{$\begin{array}{c}\text { Average Velocity }(\mathrm{m} / \mathrm{s}) \\
\mathrm{d}=20 \mathrm{~nm}\end{array}$} \\
\hline & & $\mathrm{P}=0,5$ & $\mathrm{P}=1$ & $\mathrm{P}=0,5$ & $\mathrm{P}=1$ \\
\hline 1. & $5 \times 10^{10}$ & 0 & 5,56 & 0 & 5,09 \\
2. & $10 \times 10^{10}$ & 3,71 & 6,67 & 3,09 & 5,82 \\
3. & $15 \times 10^{10}$ & 4,55 & 7,64 & 4,00 & 6,91 \\
4. & $20 \times 10^{10}$ & 5,64 & 9,46 & 5,27 & 8,17 \\
\hline
\end{tabular}

Based on the average velocity data in the table, it can be seen that when the polarization of a given material is the same, at different nanowire diameters, the average velocity of the movement of the domain walls along with the increasing of the injected electric current density is greater. However, the average velocity of the movement of the domain walls when given the same polarization, as the nanowire diameter increases, the average velocity of the domain walls' movement will decrease. When a given nanowire diameter is the same, at different polarization of materials, the average velocity of the domain walls' movement, along with the increasing of the given electric current density, is greater. However, the average velocity of the domain Walls movement when treated giving polarization of different material at the same diameter, the greater the polarization is the greater speed of the domain Walls movement. The increasing speed of the movement of the domain walls shows, the faster the resulting magnetization process. In this case, the material's polarization is directly proportional to the magnetization of the material [11]. The polarization factor of this material contributes significantly to the magnitude of the velocity of the domain walls' movement.

\section{Conclusions}

Based on the discussion of the results of research regarding the study of phenomenon STT on permalloy NiFe material, it can be seen that the injected electric current density can affect the magnetization value of the material. The effect given can be seen based on the domain wall's 
position, the structure of the domain wall, and the magnetization graph against time (M-t). The total energy in a ferromagnetic system can be affected by the size of the nanowire diameter. The effect given is that the larger the nanowire diameter is in the polarization of the same material, the total energy will increase, the exchange energy decreases, and the demagnetization energy increases with increasing electric current density variations. The domain wall's speed is influenced by the increasing variety of electric current density, but the polarization of a given material also affects the speed. The greater the polarization applied to the same diameter, the greater the movement of the domain walls.

\section{References}

[1] S A Wolf, D D Awschalom and R A Buhrman, 2001, Spintronics: a spin-based electronics vision for the future, Journal of Physic, Volume 294 (5546), page 1488-1495.

[2] Ismail, 2013, Studi Mikromagnetik Proses Magnetisasi dan Spektrum Suseptibilitas Ferromagnetik Elemen Diamond-Spaped, Tesis, Depok: Universitas Indonesia.

[3] T L Gerretsen, 2008, Spin Transfer Torque in Ferromagnetic Materials, Journal of Physic, Volume 8(1), page 1- 8.

[4] R A Putu, 2010, Bahan Isolasi Keramik, Skripsi, Denpasar: Fakultas Tekhnik Universitas Udayana.

[5] R D Michael and M J Donahue, 1997, Head to head domain walls in soft nano-strips: a refined phase diagram, Journal of Physic, Volume 3(1), page 1-3.

[6] Y Nakatani, A Thiaville, J Miltat and N Vernier, 2004, Domain wall motion by spin-polarized current: a micromagnetic study. Journal of Physics, Volume 95(1), page 1-5.

[7] V Vlaminck and M Bailleul, 2008, Current-induced Spin Wave Doppler Shift, Journal of Physics, Volume 322(1), page 410- 413.

[8] M Franchin, 2009, Multiphysics Simulation of Magnetic Nanostructures, Thesis, Malaysia: University of Southampton.

[9] T M Atmono and Yunanto, 2002, Efek Giant Magnetoresistance pada Logam Peralihan Co, $\mathrm{Ni}, \mathrm{Cu}$, Jurnal Sains, Volume 6(1), page 58.

[10] Mardona, 2012, Dinamika Domain Wall dan Efek Anisotropi pada Material Ferromagnet Co dan Ni Berbentuk Nanowire, Tesis, Jakarta: Universitas Jakarta.

[11] G Bertoti, 1998, Hysteresis in magnetism, New York: Academic Press. 